\title{
Transposable elements and gene expression during the evolution of amniotes
}

\author{
Lu Zeng ${ }^{1}$, Stephen M. Pederson², R.Daniel Kortschak ${ }^{1}$ and David L. Adelson ${ }^{*}$ (1)
}

\begin{abstract}
Background: Transposable elements (TEs) are primarily responsible for the DNA losses and gains in genome sequences that occur over time within and between species. TEs themselves evolve, with clade specific LTR/ERV, LINES and SINEs responsible for the bulk of species-specific genomic features. Because TEs can contain regulatory motifs, they can be exapted as regulators of gene expression. While TE insertions can provide evolutionary novelty for the regulation of gene expression, their overall impact on the evolution of gene expression is unclear. Previous investigators have shown that tissue specific gene expression in amniotes is more similar across species than within species, supporting the existence of conserved developmental gene regulation. In order to understand how species-specific TE insertions might affect the evolution/conservation of gene expression, we have looked at the association of gene expression in six tissues with TE insertions in six representative amniote genomes.

Results: A novel bootstrapping approach has been used to minimise the conflation of effects of repeat types on gene expression. We compared the expression of orthologs containing recent TE insertions to orthologs that contained older TE insertions, and the expression of non-orthologs containing recent TE insertions to non-orthologs with older TE insertions. Both orthologs and non-orthologs showed significant differences in gene expression associated with TE insertions. TEs were found associated with species-specific changes in gene expression, and the magnitude and direction of expression changes were noteworthy. Overall, orthologs containing species-specific TEs were associated with lower gene expression, while in non-orthologs, non-species specific TEs were associated with higher gene expression. Exceptions were SINE elements in human and chicken, which had an opposite association with gene expression compared to other species.
\end{abstract}

Conclusions: Our observed species-specific associations of TEs with gene expression support a role for TEs in speciation/response to selection by species. TEs do not exhibit consistent associations with gene expression and observed associations can vary depending on the age of TE insertions. Based on these observations, it would be prudent to refrain from extrapolating these and previously reported associations to distantly related species.

Keywords: Transposon, Gene expression, Amniotes, Evolution, Retrotransposon

\section{Background}

Transposable Elements (TEs) have been shown to alter gene regulation and drive genome evolution [1-5]. TEs can exert these effects on genes by altering chromatin structure, providing novel promoters or insulators, novel splice sites or other post-transcriptional modifications to re-wire transcriptional networks important in development and reproduction $[3,6]$. TEs that land in introns can

${ }^{*}$ Correspondence: david.adelson@adelaide.edu.au

${ }^{1}$ School of Biological Sciences, The University of Adelaide, North Terrace, 5005 Adelaide, Australia

Full list of author information is available at the end of the article become "exonized" or spliced into mRNA of the gene into which they have inserted, often introducing stop codons into mRNA that can lead to nonsense-mediated mRNA decay, serving to control gene expression $[7,8]$.

Short INterspersed Elements (SINEs) are non-autonomous TEs ancestrally related to functionally important RNAs, such as tRNA, 5S rRNA and 7SL RNA that replicate by retrotransposition. SINEs possess an internal promoter that can be recognized and transcribed by the RNA polymerase III (polIII) enzyme complex, and are usually present in a monomeric or tandem dimeric structure [9]. Monomeric tRNA-related SINE families are present

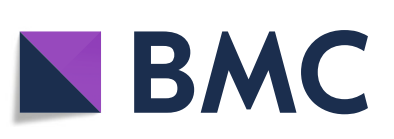

(c) The Author(s). 2018 Open Access This article is distributed under the terms of the Creative Commons Attribution 4.0 International License (http://creativecommons.org/licenses/by/4.0/), which permits unrestricted use, distribution, and reproduction in any medium, provided you give appropriate credit to the original author(s) and the source, provide a link to the Creative Commons license, and indicate if changes were made. The Creative Commons Public Domain Dedication waiver (http://creativecommons.org/publicdomain/zero/1.0/) applies to the data made available in this article, unless otherwise stated. 
in the genomes of species from all major eukaryotic lineages and this structure is, by far, the most frequent. These elements are composed of a 5' tRNA-related region and a central region of unknown origin, followed by a stretch of homopolymeric adenosine residues or other simple repeats $[10,11]$. In contrast to the very widespread phylogenetic distribution of tRNA derived SINEs, 7SLderived SINEs have been found only in mammals [9]. They are composed of a 7SL-derived region followed by a poly(A) tail and can be either monomeric (B1 family) or dimeric (Alu family) [12, 13]. 5S rRNA-derived SINEs were found in fishes (SINE3) but were likely active in the common ancestor of vertebrates $[14,15]$. They are with a 5S-related region (instead of a tRNA-related region), followed by a central region of unknown origin and 3'terminal repeats [14]. SINE RNAs have also been shown to possess the potential to regulate gene expression at the post-transcriptional level, for example, Alu RNAs can modulate protein translation, influence on RNA editing and mRNA splicing [16].

Long INterspersed Elements (LINEs) are autonomously replicating TEs that replicate through an RNA intermediate that is reverse transcribed back into the genome at a new location. LINEs contain an internal DNA Polymerase II promoter and either one or two Open Reading Frames (ORFs) that contain a Reverse Transcriptase (RT) domain and an Endonuclease (EN) domain. L1 family repeats show a stronger negative correlation with expression levels than the gene length [17], and the presence of L1 sequences within genes can lower transcriptional activity [18].

Long terminal repeat (LTR) retrotransposons are a group of TE, that are flanked by long terminal repeats and contain two ORFs: gag and pol. The gag ORF encodes the structural protein that makes up a virus-like particle [19]. The pol ORF encodes an enzyme needed for replication that contains protease, integrase, reverse transcriptase, and RNase $\mathrm{H}$ domains required for reverse transcription and integration. LTRs can also act as alternative promoters to provide new tissue-specificity, act as the major promoters, or exert only minor effects [20]. Many endogenous retroviruses (ERV) contain sequences that can serve as transcriptional start sites or as cis-acting regulatory elements in the host genomes [21].

DNA transposons encode a transposase gene that is flanked by two Terminal Inverted Repeats (TIRs) [22]. The transposase recognizes these TIRs to excise the transposon DNA, which is then inserted into a new genomic location by cut and paste mobilization[23]. DNA transposons can inactivate or alter the expression of genes by insertion within introns, exons or regulatory region $[2,22]$.

There is a growing realization that many TEs are highly conserved among distantly related taxonomic groups, suggesting their biological value to the genome. In this report, we describe the association of clade specific TEs with gene expression in long diverged amniotes (Fig. 1a) in order to determine how much these TEs might have altered the regulation of gene expression in six tissues during the evolution of these species.

\section{Methods}

\section{Expression data}

RNA-Seq expression data were available for six species (Table 1), belonging to the five main amniote lineages (eutherian: human; marsupial: gray short-tailed opossum; monotreme: platypus; lepidosaur: green anole lizard, bearded dragon; archosaur: chicken) from four somatic (brain, heart, liver, kidney) and two reproductive tissues (testis, ovary)(Gene Expression Omnibus accession numbers GSE30352 [24] and GSE97367 [25], BioProject number PRJEB5206 [26]).

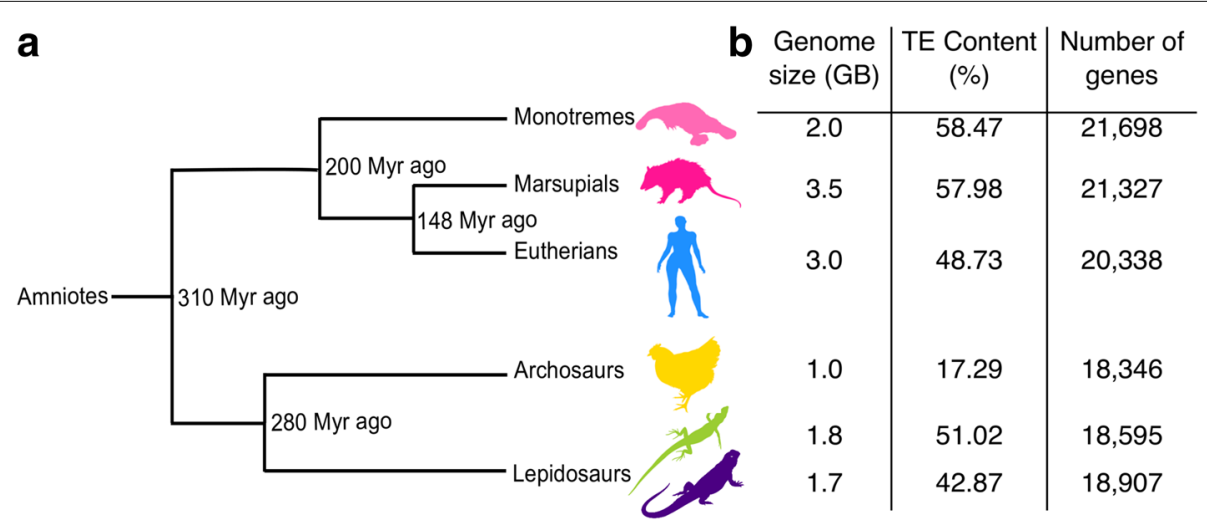

Fig. 1 Divergence times and genome statistics of the major amniote lineages. a) The silhouettes indicate species used in this study: Ornithorhynchus anatinus (platypus), Monodelphis domestica (opossum), Homo sapiens (human), Gallus gallus (chicken), Anolis carolinensis (anole lizard) and Pogona vitticeps (bearded dragon) (from top to bottom). Time since main speciation events obtained from TimeTree (www.timetree.org) are indicated (millions of years ago, Myr ago) [25]; b) Genome statistics 
Table 1 Summary of datasets and tissue samples analyzed in this study

\begin{tabular}{lll}
\hline Dataset(s) & Tissues & Species \\
\hline Marin [25] & $\begin{array}{l}\text { brain, heart, kidney, liver, } \\
\text { ovary, testes }\end{array}$ & $\begin{array}{l}\text { chicken, anole, platypus, } \\
\text { opossum, *human }\end{array}$ \\
Brawand [24] & $\begin{array}{l}\text { brain, heart, kidney, liver, } \\
\text { testes }\end{array}$ & $\begin{array}{l}\text { chicken, platypus, } \\
\text { opossum, human }\end{array}$ \\
Georges [26] & $\begin{array}{l}\text { brain, heart, kidney, liver, } \\
\text { ovary, testes }\end{array}$ & bearded dragon \\
\hline
\end{tabular}

"Human samples in this set do not include ovary tissue

Trim_galore (v0.4.2)(-clip_R1 5; -three_prime_clip_R1 5) [27] was used for adapter trimming and quality control. Adapter-trimmed RNA-Seq reads were aligned to the reference genomes (Ensembl release 74) with RSEM (v1.3.0) [28] using Bowtie2 (v2.2.9) [29] with default parameters as the alignment tool. Gene expression was estimated as TPM (Transcripts Per Million). A complete list of accessions can be found in Additional file 1: Table S1.

\section{Genomic data}

For chicken, anole lizard, platypus, opossum and human, gene annotations were download from Ensembl release 74. For bearded dragon, RefSeq assembly GCF_900067755.1 was used for analysis. Complete information on genomes used can be found in Additional file 1: Table S2.

\section{Ortholog definition}

Gene orthologies were downloaded from Ensembl release 74. Amniote orthologs were defined as single-copy orthologous genes conserved in all 6 amniote species. Reciprocal best hits were used to extract orthologous genes between bearded dragon and other five species by using BLASTN [30]. A total number of 6595 orthologous genes were extracted from the six species.

\section{TE annotation}

TEs were annotated by using CARP: an ab initio method [31]. Recently inserted, low divergence TE referred to hereafter as species-specific TE (ssTE) were defined as having $\geq 94 \%$ sequence identity, and are considered recently active. They were extracted from CARP output, which identifies and annotates TEs that have $\geq 94 \%$ sequence identity. Older TEs were defined as the remaining TE insertions in the genome and are referred to as non-species specific TE (nsTE).

\section{Clustering and distance metrics}

Three clustering methods were used to visualize and identify transcriptome clusters from the amniote tissue dataset. The first method is a standard approach to clustering; Principal component analysis (PCA) [32]. The central idea of PCA is to reduce the dimensionality of the data set while retaining as much of the variation as possible in order to identify the principal components of the variance. In addition, Unweighted Pair Group Method with Arithmetic Mean (UPGMA) [33] and Ward's minimum variance (ward.D2) [34] methods were used as alternatives to visualize and identify transcriptome clusters. UPGMA is an agglomerative hierarchical clustering algorithm yielding a dendrogram that can be cut at a chosen height to produce the desired number of clusters $(-$ cuttree $=5)$. It uses a dissimilarity matrix in order to decide if two expression profiles are close or not. Ward.D2 minimum variance method is the only agglomerative clustering method that is based on a classical sum-of-squares criterion, producing groups that minimize within-group dispersion at each binary fusion, and it identifies clusters in multivariate Euclidean space (-cuttree=7).

\section{The weighted bootstrap procedure for assessing association of gene expression and TEs}

Many genes contain multiple transposable elements, with only a minority of genes containing a single TE. In order to assess any effects on transcription due to the presence of a single TE, a weighted bootstrap approach was devised. Bootstrapping is a statistical technique using random sampling methods [35]. It samples a population, measures a statistic for this sample, repeats this step many times, and then uses this statistic to estimate the corresponding parameter of the population.

For a given TE type within each individual gene, the frequencies of co-occurring TE types and combinations of TE types were noted (Fig. 3). Uniform sampling probabilities were then used for the set of genes containing a specific TE type (test sample), whilst sampling weights were assigned to genes lacking the specific TE type based on TE composition (reference sample) (See detail in Additional file 1: Table S3-7). Gene length was divided into 10 bins and these were included as an additional category when defining sampling weights. This ensured that two gene sets were obtained for each bootstrap iteration, which were matched in length and TE composition with the sole difference being the presence of the specific TE type. The median difference in expression level, as measured by $\log 2(\mathrm{TPM})$, and the difference in the proportions of genes detected as expressed were then used as the variables of interest in the bootstrap procedure. The bootstrap was performed on sets of 1000 genes (except for ortholog genes containing non-species specific SINE elements in platypus) for 5000 iterations. Samples that could not meet the minimum number of 600 genes were not used. When comparing expression levels, genes with zero read counts were omitted prior to bootstrapping. In order to compensate for multiple testing considerations, confidence intervals were obtained across the $\mathrm{m}=$ nTissues ${ }^{*} \mathrm{nElements}$ tests at the level $1-\alpha / \mathrm{m}$, giving confidence intervals that controlled the family-wise 
error rate at the level $\alpha=0.05$. Approximate two-sided $p$ values were also calculated by finding the point at which each confidence interval crossed zero, and additional significance was determined by estimating the FDR on these sets of $p$-values using the Benjamini-Hochberg method.

\section{Results}

\section{Mammalian gene expression phylogenies}

To obtain an initial overview of gene expression patterns, we evaluated the similarity of ortholog gene expression in 6 tissues (heart, brain, kidney, liver, testis and ovary), from both males and females in our 6 species. These RNASeq samples were assembled from three different studies (Table 1, further detail can be seen in Additional file 1: Table S1) [24-26].

Two hierarchical clustering methods were used to investigate the conservation of expression signatures in these six species within six tissues. 1 - UPGMA and 2 - ward.D2 hierarchical clustering.

While mostly similar, the two methods did give slightly different clustering results (Fig. 2). Generally, gene expression clustered according to tissue with three exceptions. The first exception was bearded dragon heart expression clustered using Ward's method, where heart samples clustered with kidney and liver samples. The second exception was for platypus testis expression clustered using UPGMA, where testis expression clustered with ovary. The third exception was more widespread, and found with both clustering methods; kidney and liver samples only clustered by tissue for human and opossum and were found together more often in species-specific clusters for the other species.

\section{Comparison of gene expression for genes on the basis of their TE content}

There were two aspects of the data that affected our analysis. First, because the vast majority of genes contain TEs, it was impossible to compare expression of genes with TEs against genes without TEs, as there were too few of the latter. So we designed our comparisons as shown in Fig. 3. Second, most genes contain multiple TE types. In order to minimize the conflation of co-occuring TEs, a weighted bootstrap approach was used in this study. The idea is simple, if we want to investigate the association between a SINE insertion and gene expression, first we randomly select 1000 genes that contain a SINE element, and then compare their expression level to 1000 randomly selected genes that do not contain any SINEs. We repeat this process 5000 times in order to generate enough observations for statistical analysis.

\section{Ortholog expression is associated with TE type}

For our specific analyses, BedTools was used to get the intersection between TE types and 6595 orthologous genes (including $1 \mathrm{~kb}$ upstream and $1 \mathrm{~kb}$ downstream regions) within our six species (chicken, anole lizard, bearded dragon, platypus, opossum and human). The boostrap approach as described above was then applied to this data in order to investigate the association between orthologous gene expression and TE insertions. TEs were split into two groups: recently inserted, low divergence TEs, referred to as species-specific TEs (ssTEs, see Methods for detail) and more divergent TEs, referred to as non-species specific TEs (nsTEs). Genes containing no TEs are referred to as $\varnothing \mathrm{TE}$. The two TE groups were further broken down into four TE classes: DNA transposon, ERV/LTR, LINE or SINE.

Because purifying selection is likely to be more common on orthologs, and since tissue specificity of ortholog expression was largely conserved (Fig. 2), we looked first at the association ortholog expression with TE insertions. We compared expression for orthologs containing ssTE against orthologs containing nsTE $+\varnothing \mathrm{TE}$ and expression of orthologs containing nsTE against orthologs containing ssTE $+\varnothing \mathrm{TE}$ (Fig. 4) and (Additional file 1: Figures S1 and S2). We found that ssTEs (ERV/LTR, LINE and SINE) were associated with lower gene expression in orthologs, especially in anole lizard, bearded dragon and human. The exceptions to this negative association were in the human and chicken genome, where recent insertions of SINEs were found associated with higher gene expression in testis and brain.

For orthologs containing nsTE (LINE or SINE) (Fig. 4, Additional file 1: Figures S1 and S3) we observed primarily a positive association with gene expression in contrast to the trend seen with ssTEs. The exceptions to this positive association were again found in the human and chicken genomes. Particularly in the chicken genome, where the insertion of non-species specific SINEs were associated with lower ortholog gene expression in multiple tissues.

Overall, species-specific TE insertions in orthologs were mainly associated with lower gene expression, while nonspecies specific TE insertions were mainly associated with higher gene expression. This is true for ERV/LTR in anole lizard, bearded dragon and human, LINE and tRNA derived SINE insertions in anole, bearded dragon, platypus and human. There are some exceptions, notably for chicken orthologs with nsTE insertions which showed an association with decreased gene expression. Perhaps the most interesting observation was that the magnitude of the effect on gene expression was quite pronounced, ranging between about -30 to $+40 \%$ changes in median gene expression values (Additional file 1: Table S8).

\section{Non-ortholog gene expression is associated with TE type} In order to explore the association of TEs in a more general context, we then expanded our analysis from orthologous genes to non-orthologous genes. 


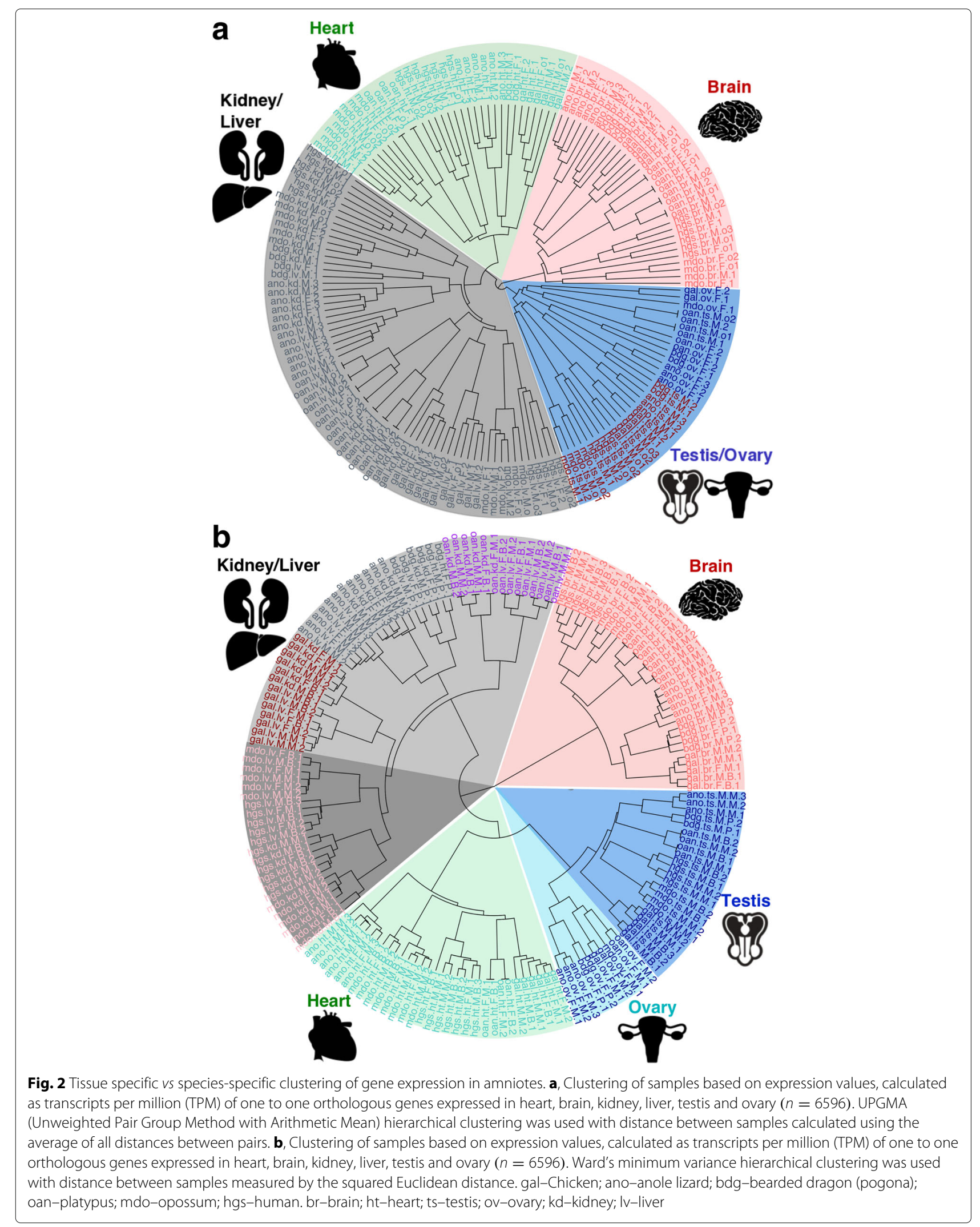




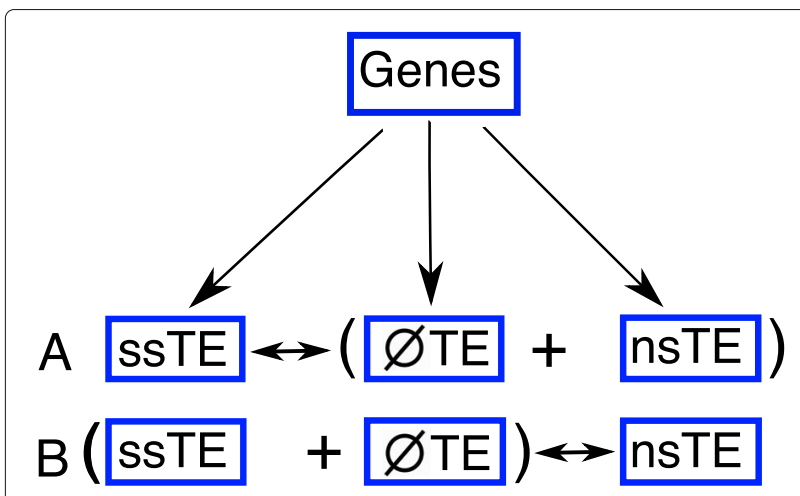

Fig. 3 Gene sets for expression comparison. Because there were too few genes (orthologs or non-orthologs) with no TE insertions, we designed comparison sets based on the following scheme. We split our gene sets (either ortholog or non-ortholog) into three subsets: those containing recent species-specific TE insertions (ssTE), those containing non-species specific TE insertions (nsTE) and those containing no TE ( $\varnothing \mathrm{TE})$

As described above, BedTools was used to get the intersection between TE types and non-orthologous genes, and the bootstrap approach was used to compare expression for non-orthologs containing ssTE against nonorthologs containing nsTE $+\varnothing \mathrm{TE}$ and expression of non-orthologs containing nsTE against non-orthologs containing ssTE $+\varnothing \mathrm{TE}$ (Fig. 4) and (Additional file 1: Figures S4 and S5).

Similar to orthologs, ssTE insertions in non-orthologs showed a negative association with gene expression. This can be observed in ERV/LTR, LINE and SINE in anole lizard and bearded dragon. In the chicken, older SINE insertions in non-orthologs were negatively associated with gene expression. In contrast to the anole lizard and bearded dragon, where recent ERV/LTR, LINE and SINE insertions were associated with lower gene expression, human (7SL derived) SINE insertions in non-orthologs were strongly associated with higher gene expression. The magnitude of the association of TEs with gene expression was even more pronounced in these comparisons, ranging from about -40 to $+180 \%$ (Fig. 4) and (Additional file 1: Figures S4 and S6, Table S8).

\section{Discussion}

Tissue $v s$ species clustering of ortholog gene expression had previously been reported using PCA based analysis and used to support the notion that conservation of developmental gene expression programs results in tissue specific gene expression clustering [24, 36, 37]. These results have been reported for single experiments. We did not see quite as compelling tissue clustering of gene expression using PCA on data from aggregated experiments (Additional file 1: Figure S8). However we did see largely similar results when we applied hierarchical clustering methods across the aggregated data (Fig. 2). However, in contrast with previous studies, we found liver and kidney gene expression clustered more by species. We attribute this to species-specific metabolic adaptations responding to more pronounced environmental selection. We expected to see species-specific TE insertions associated with species-specific changes in gene expression. For recent species-specific SINE, ERV/LTR and LINE insertions this is precisely what we found. However, we found no tissue specific patterns of association of gene expression with TEs.

We expected species-specific TE insertions to be associated with changed gene expression, as they would both alter the spacing of pre-existing regulatory motifs and potentially contribute new regulatory motifs [6, 38, 39]. Because random changes in complex systems usually break things, we expected recent TE insertions to be associated with lower gene expression. While this expectation was largely met, there were some significant exceptions, such as human SINE, which were associated with increased gene expression (see "Discussion" below). Conversely, it has been shown that older TE insertions contribute to re-wiring of transcriptional networks $[40,41]$ and thus would have had time to be exapted as enhancers and might be associated with increased gene expression. Previous studies have found that differential decay of ancestral TE sequences across species may result in species-specific transcription factor binding sites [42]. This expectation was also met for human ERV/LTR. However to our surprise, older TE SINE insertions in the chicken were associated with decreased gene expression.

We expected the magnitude of changes in gene expression associated with TE insertions to be modest, however our analysis showed that TE insertions were associated with large changes in gene expression. Based on the median value of changed gene expression from our bootstrap analysis, most statistically significant $\log 2$ transformed changes in gene expression associated with TE were smaller than -0.5 and many were greater than 1.0 , indicating a range of -40 to $+100 \%$ change in median gene expression.

Species-specific TE, behaved differently depending on insertion age and species. The most striking example of this was seen in human with recent SINE insertions associated with increased gene expression and older SINE associated with decreased gene expression. This is consistent with observations that Alu elements have been exapted as transcription factor binding sites, and highly and broadly expressed housekeeping genes are enriched for Alus [43-45]. This was in contrast to an opposite relationship with LINE insertion age and expression change in human, but consistent with previously reported accumulation differences for SINE and LINE insertions in mammalian regulatory regions/open chromatin [46]. 


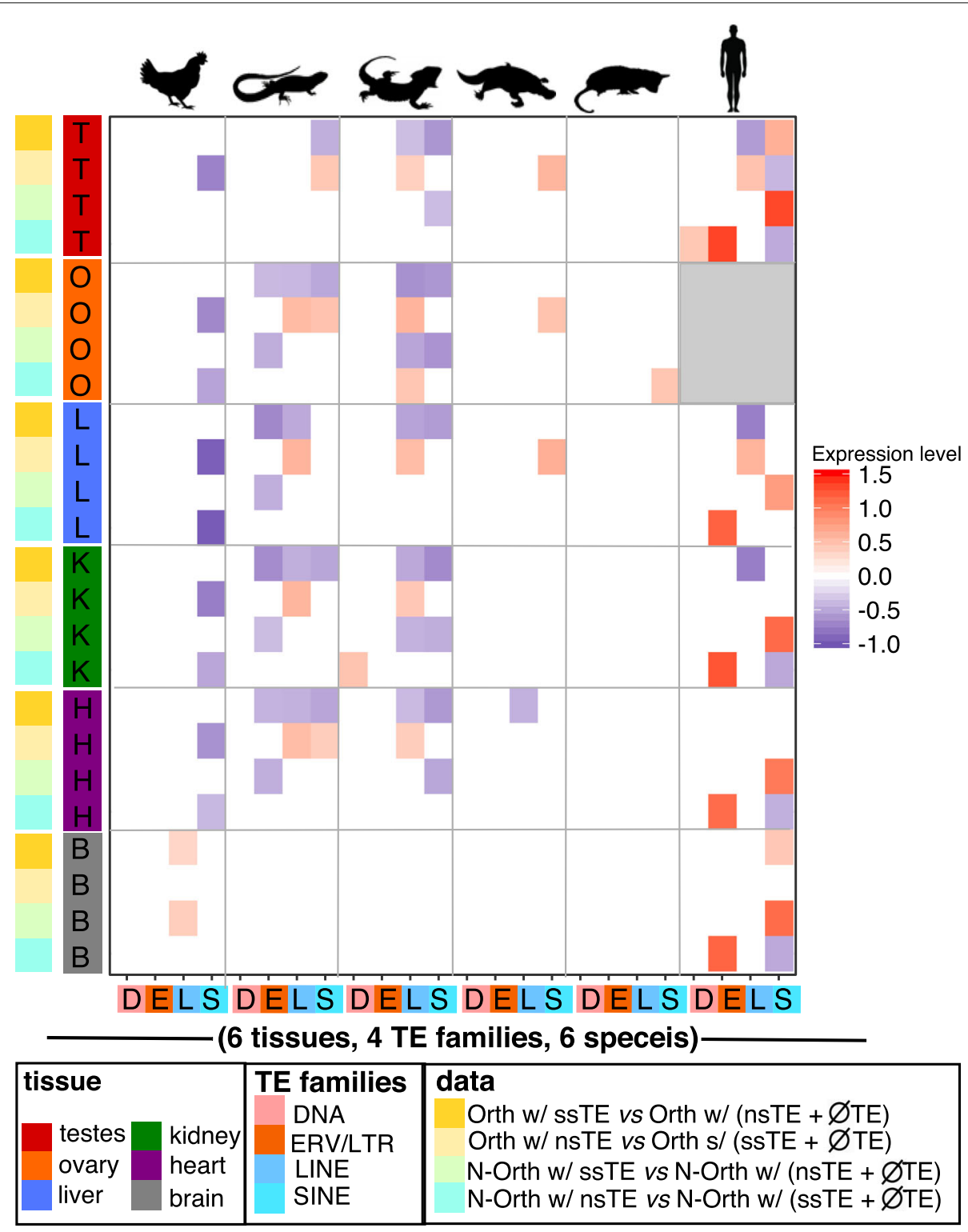

Fig. 4 Changes in the levels of ortholog/non-ortholog gene expression as a function of TE insertion. This figure shows the association between orthologous/non-orthologous gene expression levels in six species (from left to right: chicken, anole lizard, bearded dragon (pogona), platypus, opossum and human) with the presence of recent species-specific TE insertions (ssTE) or non-species specific TE insertions (nsTE) (from left to right: DNA, ERV/LTR, LINE or SINE). A weighted bootstrap approach was used to compare the median difference in gene expression levels of orthologous/non-orthologous genes with a ssTE/nsTE insertion compared to orthologous/non-orthologous gene without ssTE/nsTE. Gene expression levels are log2-transformed. Comparisons without statistically significant gene expression changes are shown in white. Statistically significant increased gene expression shown in red and statistically significant decreased gene expression in blue. Grey shading indicates no samples were available for this comparison

Furthermore, LINEs behave similarly in reptiles and human, with new LINEs associated with lower gene expression and older LINEs associated with higher gene expression. This suggests similar constraints on accumulation of TE in lizards and mammals. Finally, TEs had the fewest associations with gene expression in opossum and platypus. This might indicate that these two species are better at repressing TE activity than human, lizards and chicken.

\section{Conclusions}

The large changes in gene expression associated with TEs, and the species-specific associations of TEs with gene expression support a role for TEs in speciation/response 
to selection by species. TE types do not exhibit consistent associations with gene expression and observed associations can vary depending on the age of TE insertions. Based on these observations, it would be prudent to refrain from extrapolating these and previously reported associations to distantly related species.

\section{Additional file}

Additional file 1: Supplementary Information Additional file contains supplementary figures and tables as referred to in the main body of the paper. (PDF 848 kb)

\section{Abbreviations}

EN: Endonuclease; ERV: Endogenous retroviruses; LINE(s): Long INterspersed element(s); LTR: Long terminal repeat; nsTE(s): non-species specific TE(s); ORFs: Open reading frames; pollll: polymerase III; RT: Reverse Transcriptase; SINE(s): Short INterspersed Element(s); ssTE(s): species-specific TE(s); TE(s): Transposable element(s); TIRs: Terminal inverted repeats; TPM: Transcripts per million; UPGMA: Unweighted pair group method with arithmetic mean; vs: versus; ward.D2: Ward's minimum variance; $\varnothing T E$ : genes containing no TEs

\section{Acknowledgements}

We would like to thank Terry Bertozzi and Catisha Coburn for taking the time to read the manuscript in full and offer helpful comments. This paper would not be possible without helpful discussions with Zhipeng Qu, and extraordinary HPC support from Matt Westlake.

\section{Funding}

The University of Adelaide funded this research.

\section{Availability of data and materials}

All data analyzed in this study were accessed from the public source NCBI (https://www.ncbi.nlm.nih.gov/) and details are shown in the supplementary file. Repeat annotations and TPM values for each species will be provided upon request.

\section{Authors' contributions}

LZ, SMP, RDK, and DLA designed research; LZ and SMP performed research; and $L Z, R D K$, and DLA wrote the paper. All authors read and approved the final manuscript.

\section{Ethics approval and consent to participate}

Not applicable.

\section{Competing interests}

The authors declare that they have no competing interests.

\section{Publisher's Note}

Springer Nature remains neutral with regard to jurisdictional claims in published maps and institutional affiliations.

\section{Author details}

${ }^{1}$ School of Biological Sciences, The University of Adelaide, North Terrace, 5005 Adelaide, Australia. ${ }^{2}$ Bioinformatics Hub, The University of Adelaide, North Terrace, 5005 Adelaide, Australia.

Received: 7 March 2018 Accepted: 1 June 2018

Published online: 12 June 2018

\section{References}

1. Kapusta A, Suh A, Feschotte C. Dynamics of genome size evolution in birds and mammals. Proc Natl Acad Sci. 2017;114(8):1460-9.

2. Kazazian HH. Mobile elements: drivers of genome evolution. Science. 2004;303(5664):1626-32.

3. Cordaux R, Batzer MA. The impact of retrotransposons on human genome evolution. Nat Rev Genet. 2009;10(10):691.
4. Ponicsan SL, Kugel JF, Goodrich JA. Genomic gems: Sine rnas regulate mrna production. Curr Opin Genet Dev. 2010;20(2):149-55.

5. Buckley RM, Adelson DL. Mammalian genome evolution as a result of epigenetic regulation of transposable elements. Biomol Concepts. 2014;5(3):183-94.

6. Feschotte C. Transposable elements and the evolution of regulatory networks. Nat Rev Genet. 2008;9(5):397.

7. Ni JZ, Grate L, Donohue JP, Preston C, Nobida N, O'Brien G, Shiue L, Clark TA, Blume JE, Ares M. Ultraconserved elements are associated with homeostatic control of splicing regulators by alternative splicing and nonsense-mediated decay. Genes Dev. 2007;21(6):708-18.

8. Attig J, de los Mozos IR, Haberman N, Wang Z, Emmett W, Zarnack K, et al. Splicing repression allows the gradual emergence of new alu-exons in primate evolution. Elife. 2016;5. https://doi.org/10.7554/eLife.19545.

9. Pelissier T, Bousquet-Antonelli C, Lavie L, Deragon J-M. Synthesis and processing of trna-related sine transcripts in arabidopsis thaliana. Nucleic Acids Res. 2004;32(13):3957-66.

10. Okada N. Sines. Curr Opin Genet Dev. 1991;1 (4):498-504.

11. Okada N, Hamada M, Ogiwara I, Ohshima K. Sines and lines share common 3' sequences: a review. Gene. 1997;205(1):229-43.

12. Labuda D, Sinnett D, Richer C, Deragon J-M, Striker G. Evolution of mouse b1 repeats: $7 \mathrm{~s}$ ina folding pattern conserved. J Mol Evol. 1991;32(5):405-14.

13. Sinnett $D$, Richer $C$, Deragon J-M, Labuda D. Alu rna secondary structure consists of two independent $7 \mathrm{sl}$ rna-like folding units. J Biol Chem. 1991;266(14):8675-8.

14. Kapitonov W, Jurka J. A novel class of sine elements derived from $5 \mathrm{~s} \mathrm{rrna.}$ Mol Biol Evol. 2003;20(5):694-702.

15. Nishihara H, Smit AF, Okada N. Functional noncoding sequences derived from sines in the mammalian genome. Genome Res. 2006;16(7): 864-74.

16. Häsler J, Strub K. Alu elements as regulators of gene expression. Nucleic Acids Res. 2006;34(19):5491-7.

17. Jjingo D, Huda A, Gundapuneni M, Mariño-Ramírez L, Jordan IK. Effect of the transposable element environment of human genes on gene length and expression. Genome Biol Evol. 2011;3:259-71.

18. Han JS, Szak ST, Boeke JD. Transcriptional disruption by the I1 retrotransposon and implications for mammalian transcriptomes. Nature. 2004;429(6989):268

19. Havecker ER, Gao X, Voytas DF. The diversity of Itr retrotransposons. Genome Biol. 2004;5(6):225.

20. Cohen CJ, Lock WM, Mager DL. Endogenous retroviral Itrs as promoters for human genes: a critical assessment. Gene. 2009;448(2):105-14.

21. Jern $P$, Coffin JM. Effects of retroviruses on host genome function. Annu Rev Genet. 2008:42:709-32.

22. Muñoz-López M, García-Pérez JL. Dna transposons: nature and applications in genomics. Curr genomics. 2010;11(2):115-28.

23. Skipper KA, Andersen PR, Sharma N, Mikkelsen JG. Dna transposon-based gene vehicles-scenes from an evolutionary drive. J Biomed Sci. 2013;20(1):92.

24. Brawand $D$, Soumillon $M$, Necsulea $A$, Julien $P$, Csárdi $G$, Harrigan $P$, Weier M, Liechti A, Aximu-Petri A, Kircher M, et al. The evolution of gene expression levels in mammalian organs. Nature. 2011;478(7369):343-8.

25. Marin R, Cortez D, Lamanna F, Pradeepa MM, Leushkin E, Julien P, Liechti A, Halbert J, Brüning T, Mössinger K, et al. Convergent origination of a drosophila-like dosage compensation mechanism in a reptile lineage. Genome Res. 2017;27(12):1974-87.

26. Georges A, Li Q, Lian J, O'Meally D, Deakin J, Wang Z, Zhang P, Fujita M, Patel HR, Holleley CE, et al. High-coverage sequencing and annotated assembly of the genome of the australian dragon lizard pogona vitticeps. Gigascience. 2015;4(1):45.

27. Krueger F. Trim galore. A wrapper tool around Cutadapt and FastQC to consistently apply quality and adapter trimming to FastQ files. 2015 https://www.bioinformatics.babraham.ac.uk/projects/trim_galore/.

28. Li B, Dewey CN. Rsem: accurate transcript quantification from rna-seq data with or without a reference genome. BMC Bioinformatics. 2011;12(1): 323.

29. Langmead B, Salzberg SL. Fast gapped-read alignment with bowtie 2 . Nat Methods. 2012;9(4):357.

30. Altschul SF, Gish W, Miller W, Myers EW, Lipman DJ. Basic loca alignment search tool. J Mol Biol. 1990;215(3):403-10. 
31. Zeng L, Kortschak RD, Raison JM, Bertozzi T, Adelson DL. Superior ab initio identification, annotation and characterisation of tes and segmental duplications from genome assemblies. PLoS One. 2018;13:e0193588.

32. Dunteman GH. Principal Components Analysis. United States: Sage; 1989.

33. Sneath P, Sokal RR, et al. Numerical Taxonomy. The Principles and Practice of Numerical Classification. Numerical Taxonomy. San Franciso: Freeman; 1973.

34. Murtagh F, Legendre P. Ward's hierarchical agglomerative clustering method: which algorithms implement ward's criterion? J Classif. 2014;31(3):274-95.

35. Varian H. Bootstrap tutorial. Math J. 2005;9(4):768-75.

36. Sudmant PH, Alexis MS, Burge CB. Meta-analysis of rna-seq expression data across species, tissues and studies. Genome Biol. 2015;16(1):287.

37. Merkin J, Russell C, Chen P, Burge CB. Evolutionary dynamics of gene and isoform regulation in mammalian tissues. Science. 2012;338(6114):1593-9.

38. Slotkin RK, Martienssen R. Transposable elements and the epigenetic regulation of the genome. Nat Rev Genet. 2007;8(4):272.

39. Bourque $\mathrm{G}$. Transposable elements in gene regulation and in the evolution of vertebrate genomes. Curr Opin Genet Dev. 2009;19(6): $607-12$.

40. Lynch VJ, Nnamani MC, Kapusta A, Brayer K, Plaza SL, Mazur EC, Emera D, Sheikh SZ, Grützner F, Bauersachs S, et al. Ancient transposable elements transformed the uterine regulatory landscape and transcriptome during the evolution of mammalian pregnancy. Cell Rep. 2015;10(4):551-61.

41. Rebollo R, Romanish MT, Mager DL. Transposable elements: an abundant and natural source of regulatory sequences for host genes. Annu Rev Genet. 2012;46:21-42.

42. Chuong EB, Elde NC, Feschotte C. Regulatory activities of transposable elements: from conflicts to benefits. Nat Rev Genet. 2017;18(2):71.

43. Polak P, Domany E. Alu elements contain many binding sites for transcription factors and may play a role in regulation of developmental processes. BMC Genomics. 2006;7(1):133.

44. Piedrafita FJ, Molander RB, Vansant G, Orlova EA, Pfahl M, Reynolds WF. An alu element in the myeloperoxidase promoter contains a composite sp1-thyroid hormone-retinoic acid response element. J Biol Chem. 1996;271(24):14412-20.

45. Eller CD, Regelson M, Merriman B, Nelson S, Horvath S, Marahrens Y. Repetitive sequence environment distinguishes housekeeping genes. Gene. 2007;390(1):153-65.

46. Buckley RM, Kortschak RD, Raison JM, Adelson DL. Similar evolutionary trajectories for retrotransposon accumulation in mammals. Genome Biol Evol. 2017;9(9):2336-53.

Ready to submit your research? Choose BMC and benefit from:

- fast, convenient online submission

- thorough peer review by experienced researchers in your field

- rapid publication on acceptance

- support for research data, including large and complex data types

- gold Open Access which fosters wider collaboration and increased citations

- maximum visibility for your research: over $100 \mathrm{M}$ website views per year

At BMC, research is always in progress.

Learn more biomedcentral.com/submissions 